\title{
Extremal norms of graphs and matrices
}

\author{
Vladimir Nikiforov ${ }^{* \dagger}$
}

October 24, 2018

\begin{abstract}
In the recent years, the trace norm of graphs has been extensively studied under the name of graph energy. In this paper some of this research is extended to more general matrix norms, like the Schatten $p$-norms and the Ky Fan $k$-norms. Whenever possible the results are given both for graphs and general matrices. In various contexts a puzzling fact was observed: the Schatten $p$-norms are widely different for $1 \leq p<2$ and for $p \geq 2$.
\end{abstract}

Keywords: graph energy, graph eigenvalues, singular values, matrix energy, Wigner's semicircle law

\section{Introduction}

The aim of this paper is twofold: first, to study the Schatten and the Ky Fan norms of the adjacency matrices of graphs, and second, obtain extremal properties of these norms starting from graph results. We shall show that some well-known problems and results in spectral graph theory are, in fact, best stated in terms of matrix norms. Moreover, when stated in such terms, graph theoretical proofs may be extended to arbitrary matrices, thus providing extremal relations about matrix norms.

Let $G$ be a graph of order $n$ and and let $\mu_{1}(G) \geq \cdots \geq \mu_{n}(G)$ be the eigenvalues of its adjacency matrix $A(G)$. Gutman [11, 12] introduced the energy $\mathcal{E}(G)$ of $G$ as

$$
\mathcal{E}(G)=\left|\mu_{1}\right|+\cdots+\left|\mu_{n}\right| .
$$

This parameter, initially conceived for applications in chemistry, recently got huge attention for its own sake: more than hundred papers have been devoted to the study $\mathcal{E}(G)$ and its variants. In particular, in [21], graph energy has been generalized to arbitrary matrices, including nonsquare ones: for an $m \times n$ matrix $A$, define the energy $\mathcal{E}(A)$ of $A$ as

$$
\mathcal{E}(A)=\sigma_{1}(A)+\cdots+\sigma_{m}(A),
$$

${ }^{*}$ Department of Mathematical Sciences, University of Memphis, Memphis TN 38152, USA

${ }^{\dagger}$ Research supported by NSF Grant DMS-0906634. 
where $\sigma_{1}(A) \geq \sigma_{2}(A) \geq \cdots$ are the singular values of $A$, that is to say, the square roots of the eigenvalues of $A A^{*}$, and $A^{*}$ is the Hermitian adjoint of $A$.

Since an $m \times n$ matrix $A$ has at most $\min (m, n)$ nonzero singular values, $\mathcal{E}(A)$ is just the sum of all singular values of $A$. Note also that the singular values of a Hermitian matrix are the moduli of its eigenvalues, and so, if $A$ is the adjacency matrix of a graph $G$, then $\mathcal{E}(A)=\mathcal{E}(G)$; therefore, $\mathcal{E}(A)$ indeed generalizes the concept of graph energy. Moreover, the definition (1) sheds new light on this concept since the sum of the singular values of $A$ is the well-studied trace or nuclear norm of $A$. It seems that graph energy is interesting precisely because the trace norm is a fundamental matrix parameter anyway .

Although viewing $\mathcal{E}(G)$ as the trace norm of $A(G)$ proved to be useful (see, e.g., [4], [5] and 24] for some applications), this direction was not investigated much further. To outline a new line of possible research, we first introduce some notation and definitions.

We write $\mathcal{M}_{m, n}$ for the set of complex matrices of size $m \times n$. Given a matrix $A=\left[a_{i j}\right] \in \mathcal{M}_{m, n}$, we write $|A|_{\infty}$ for $\max _{i, j}\left|a_{i j}\right|$, and $|A|_{p}$ for $\left(\sum_{i, j}\left|a_{i j}\right|^{p}\right)^{1 / p}$.

Now, let us recall the definitions of the Schatten and the Ky Fan matrix norms:

Definition 1 Given $A \in \mathcal{M}_{m, n}$ and $p \geq 1$, the Schatten $p$-norm $\|A\|_{S_{p}}$ is given by

$$
\|A\|_{S_{p}}=\left(\sigma_{1}^{p}(A)+\cdots+\sigma_{m}^{p}(A)\right)^{1 / p} .
$$

Definition 2 Given an integer $k \geq 1$, the Ky Fan $k$-norm $\|A\|_{F_{k}}$ is given by

$$
\|A\|_{F_{k}}=\sigma_{1}(A)+\cdots+\sigma_{k}(A) .
$$

If $G$ is a graph with adjacency matrix $A$, for short we write $\|G\|_{S_{p}},\|G\|_{F_{k}}$ and $|G|_{p}$ for $\|A\|_{S_{p}}$, $\|A\|_{F_{k}}$ and $|A|_{p}$, respectively. Clearly, since $\|G\|_{S_{1}}=\|G\|_{F_{n}}=\mathcal{E}(G)$, both $\|G\|_{S_{p}}$ and $\|G\|_{F_{k}}$ extend $\mathcal{E}(G)$. Throughout this paper we adopt $\|G\|_{S_{1}}$ and $\|A\|_{S_{1}}$ for $\mathcal{E}(G)$ and $\mathcal{E}(A)$.

Note that, in addition to graph energy, for positive integers $k$, the norms $\|G\|_{S_{2 k}}$ are in wide albeit implicit use in graph theory. Indeed, using the well-known fact about any matrix $A$

$$
\|A\|_{S_{2}}^{2}=\sigma_{1}^{2}(A)+\cdots+\sigma_{m}^{2}(A)=\operatorname{tr}\left(A A^{*}\right)=|A|_{2}^{2},
$$

for graphs we find that

$$
\|G\|_{S_{2}}=\sqrt{2 e(G)}
$$

where, as usual, $e(G)$ stands for the number of edges of $G$. More generally, if $k$ is a positive integer and $G$ is a graph with adjacency matrix $A$, then

$$
\|G\|_{S_{2 k}}=\left(\operatorname{tr}\left(A^{2 k}\right)\right)^{1 / 2 k}
$$

and so, $\|G\|_{S_{2 k}}^{2 k}$ is just the number of closed walks of length $2 k$ in $G$ - a well studied graph parameter.

Since particular Schatten and Ky Fan norms are already used implicitly in graph theory, we suggest an explicit and focused study of $\|G\|_{S_{p}}$ and $\|G\|_{F_{k}}$ for arbitrary $p$ and $k$. In particular, the following general problem seems interesting: 
Problem 3 Study the extrema of $\|G\|_{S_{p}}$ and $\|G\|_{F_{k}}$, and their relations to the structure of $G$.

Following this research line, below we extend some basic results about graph energy and other related topics.

On the other hand, many sound results in spectral graph theory can be readily extended to matrices, sometimes even to nonsquare ones. This fact prompts another line of investigation:

Problem 4 Adopting techniques from graph theory, study extremal properties of $\|A\|_{S_{p}}$ and $\|A\|_{F_{k}}$, and their relation to the structure of $A$ when $A$ belongs to a given class of matrices.

We give a few such results in Section [3, but they are just the tip of the iceberg. One general finding is that, in various contexts, Schatten $p$-norms are widely different for $1 \leq p<2$ and for $p \geq 2$; we have no explanation of this fact.

The rest of the paper is organized as follows: in Section 2 we discuss extremal results about the Schatten norms and Ky Fan norms of graphs: We first find the Schatten $p$-norms of almost all graphs, which turn out to be highly concentrated; then we extend McClelland's inequality [18] and some results of Koolen and Moulton [17]. We also extend a lower bound due to Caporossi, Cvetković, Gutman and Hansen [3], and a recent result of Mohar [19] on the sum of the largest eigenvalues of a graph. In Section 3 we extend some of the results in Section 2 to matrices as general as possible. At the end we outline some open problems.

We do not give detailed proofs of all stated results, but we will give enough clues to complete the proofs or to find them elsewhere.

\section{Extremal results about Schatten and Ky Fan norms of graphs}

For undefined graph theoretic and matrix notation we refer the reader to [2] and [16].

Our first topic is intended to provide some intuition what are the Schatten $p$-norms of the "average" graph.

\subsection{The Schatten norms of almost all graphs}

The purpose here is to find $\|G\|_{S_{p}}$ for all $p \geq 1$ and almost all graphs $G$. We shall prove the following theorem.

Theorem 5 If $G$ is a a graph of order $n$, then with probability tending to 1,

$$
\|G\|_{S_{p}}= \begin{cases}\left(\frac{1}{\sqrt{\pi}} \cdot \frac{\Gamma(p / 2+1 / 2)}{\Gamma(p / 2+2)}+o(1)\right)^{1 / p} n^{1 / p+1 / 2} & \text { if } 1 \leq p<2 \\ (1 / \sqrt{2}+o(1)) n & \text { if } p=2 \\ (1 / 2+o(1)) n & \text { if } p>2 .\end{cases}
$$


Proof It is clear that the Schatten $p$-norms of almost graphs can be reduced to finding the Schatten $p$-norms of the random graph $G(n, 1 / 2)$, which turn out to be highly concentrated. Thus, for our calculations we will need some results about random matrices. For short we shall write a.s. instead of "with probability tending to 1". Recall that the adjacency matrix $A(n)$ of the random graph $G(n, 1 / 2)$ is a random symmetric matrix with zero diagonal, whose entries $a_{i j}$ are independent random variables with mean $\mathbb{E}\left(a_{i j}\right)=1 / 2$, variance $\operatorname{Var}\left(a_{i j}^{2}\right)=1 / 4$, and $\mathbb{E}\left(a_{i j}^{2 k}\right)=1 / 4^{k}$ for all $1 \leq i<j \leq n, k \geq 1$. The result of Füredi and Komlós [9] implies that, a.s.

$$
\begin{aligned}
& \sigma_{1}(A(n))=(1 / 2+o(1)) n, \\
& \sigma_{2}(A(n)) \leq(1+o(1)) n^{1 / 2} .
\end{aligned}
$$

In finding $\|A(n)\|_{S_{p}}$, we shall distinguish three cases: $p>2, p=2$, and $1 \leq p<2$. Let first $p>2$. For every graph $G$ of order $n$ we have

$$
\sigma_{1}^{p}(G) \leq\|G\|_{S_{p}}^{p} \leq \sigma_{1}^{p}(G)+n \sigma_{2}^{p}(G) .
$$

By (2) and (3), we see that a.s.

$$
\left(2^{-p}+o(1)\right) n^{p} \leq\|A(n)\|_{S_{p}}^{p} \leq\left(2^{-p}+o(1)\right) n^{p}+O\left(n^{1+p / 2}\right),
$$

Thus, we see that, a.s.,

$$
\|A(n)\|_{S_{p}}=(1 / 2+o(1)) n .
$$

When $1 \leq p<2$ we apply Wigner's semicircle law [25] in the form given by Arnold [1], p. 263. Given the conditions on $A(n)$, and the fact that $1 \leq p<2$, one can show that a. s.

$$
\begin{aligned}
\|A(n)\|_{S_{p}}^{p}-\sigma_{1}^{p}(A(n)) & =n^{p / 2+1}\left(\frac{2}{\pi} \int_{-1}^{1}|x|^{p} \sqrt{1-x^{2}} d x+o(1)\right) \\
& =\frac{4 n}{\pi}\left(\int_{0}^{1} x^{p} \sqrt{1-x^{2}} d x+o(1)\right) .
\end{aligned}
$$

Here we use that the order of $\|A(n)\|_{S_{p}}^{p}$ is $n^{p / 2+1}$ and all singular values of $A(n)$ except the largest one are bounded by (3) ; thus, the contribution of $\sigma_{1}(A(n))$ is negligible and so is the contribution of a vanishing proportion of any other singular values of $A$.

Using [15], p. 196, we see that

$$
\int_{0}^{1} x^{p} \sqrt{1-x^{2}} d x=\frac{\sqrt{\pi}}{4} \cdot \frac{\Gamma(p / 2+1 / 2)}{\Gamma(p / 2+2)},
$$

and therefore, a.s., if $1 \leq p<2$, we have

$$
\|A(n)\|_{S_{p}}=\left(\frac{1}{\sqrt{\pi}} \cdot \frac{\Gamma(p / 2+1 / 2)}{\Gamma(p / 2+2)}+o(1)\right)^{1 / p} n^{1 / p+1 / 2} .
$$


For $p=2$, it is a well-known fact that a.s. the random graph $G(n, 1 / 2)$ has $(1 / 4+o(1)) n^{2}$ edges and so, a.s.,

$$
\|A(n)\|_{S_{2}}^{2}=(1 / 2+o(1)) n^{2} .
$$

This completes the proof of the theorem.

In particular, for $p=1$, we see that the energy of almost all graphs of order $n$ is a.s.

$$
\left(\frac{4}{3 \pi}+o(1)\right) n^{3 / 2}
$$

We established this basic fact in [21].

Recently Du et al. [6, 7] calculated the energy of the random graph $G(n, p)$ for $p \neq 1 / 2$. However, this exercise does not generalize the above result, as these authors claim. In fact, such claim makes no sense since graph energy is concentrated around (4) and cannot take another value.

Next we investigate upper and lower bounds on the Schatten $p$-norms for any graph.

\subsection{Extremal Schatten norms of graphs}

The material in this section is by no means exhaustive: our goal is to extend just a few of the most important bounds on graph energy.

Let $G$ is a graph of order $n$ and size $m$. A simple, yet rather efficient upper bound on graph energy gives the MClelland inequality [18]

$$
\|G\|_{S_{1}} \leq \sqrt{2 m n}
$$

Using the Cauchy-Schwarz inequality, we easily generalize this bound for $\|G\|_{S_{p}}$ when $p>1$. Note, however, that the generalizations are different for $p \leq 2$ and $p>2$.

Proposition 6 If $G$ is a graph of order $n$ and size $m$, then

$$
\|G\|_{S_{p}} \leq n^{1-p / 2}(2 m)^{p / 2}
$$

for $1 \leq p \leq 2$, and

$$
\|G\|_{S_{p}} \geq n^{1-p / 2}(2 m)^{p / 2}
$$

for $p>2$.

As we will see in Theorem 17, for arbitrary matrices these bounds are as good as one can get, but for graphs there are improvements, some of which are discussed below.

\subsubsection{The maximal Schatten $p$-norms for $1 \leq p<2$}

Koolen and Moulton ([17]) proposed more sophisticated upper bounds on $\|G\|_{S_{1}}$, starting with the following one:

If $G$ is a graph of order $n$, size $m$, and spectral radius $\mu$, then

$$
\|G\|_{S_{1}} \leq \mu+(n-1)^{1 / 2}\left(2 m-\mu^{2}\right)^{1 / 2} .
$$

The straightforward proof of Koolen and Moulton in fact gives the following generalization: 
Proposition 7 If $G$ is a graph of order $n$ and size $m$ and spectral radius $\mu$, then

$$
\|G\|_{S_{p}}^{p} \leq \mu^{p}+(n-1)^{1-p / 2}\left(2 m-\mu^{2}\right)^{p / 2}
$$

for $1 \leq p \leq 2$.

Next, using that $\mu \geq 2 m / n$, we obtain another upper bound.

Theorem 8 If $1 \leq p \leq 2$ and $G$ is a graph of order $n$ and size $m \geq n / 2$, then

$$
\|G\|_{S_{p}}^{p} \leq(2 m / n)^{p}+(n-1)^{1-p / 2}\left(2 m-(2 m / n)^{2}\right)^{p / 2} .
$$

Equality holds if and only if $G$ is either $(n / 2) K_{2}$, or $K_{n}$, or a noncomplete strongly regular graph whose nontrivial eigenvalues are

$$
\pm\left(\frac{2 m-(2 m / n)^{2}}{n-1}\right)^{1 / 2} \text {. }
$$

Now, we immediately see that

$$
(2 m / n)^{p}+(n-1)^{1-p / 2}\left(2 m-(2 m / n)^{2}\right)^{p / 2}<n^{p}+n^{1-p / 2} n^{p} 2^{-p} ;
$$

hence the following assertion.

Theorem 9 If $1 \leq p<2$ and $G$ is a graph of order $n$, then

$$
\|G\|_{S_{p}}^{p} \leq 2^{-p} n^{1+p / 2}+n^{p} .
$$

This bound is tight: for all $n$ there is a graph $G$ of order $n$ satisfying

$$
\|G\|_{S_{p}}^{p}=\left(2^{-p}+o(1)\right) n^{1+p / 2} .
$$

To see the validity of the last statement, consider Paley graphs for prime $n=1(\bmod 4)$. For any other $n$ we add isolated vertices to the Paley graph of largest order less than $n$. Details of such calculations can be found in [21].

By a more careful optimization Koolen and Moulton obtained the following precise absolute bound on the energy of an $n$-vertex graph: For every graph $G$ of order $n$,

$$
\|G\|_{S_{1}} \leq \frac{n(1+\sqrt{n})}{2} .
$$

Equality is attained for strongly regular graphs with parameters

$$
(n,(n+\sqrt{n}) / 2,(n+2 \sqrt{n}) / 4,(n+2 \sqrt{n}) / 4) .
$$

For further details of these strongly regular graphs see Haemers and Xiang [13]. Finding the best approximation of $\|G\|_{S_{1}}$ for all $n$ seems a challenging open problem; a partial solution is given in 22 . 


\subsubsection{The maximal Schatten $p$-norms for $p \geq 2$}

Let $p \geq 2$ and $G$ be a graph of order $n$ and size $m$, that is to say

$$
\sigma_{1}^{2}(G)+\cdots+\sigma_{n}^{2}(G)=2 m .
$$

To maximize $\|G\|_{S_{p}}^{p}$ subject to (9), recall that the function $x^{p / 2}$ is concave for $p \geq 2$, and so, $\|G\|_{S_{p}}^{p}$ is maximal when $\sigma_{1}^{2}(G)=2 m$ and $\sigma_{2}(G)=\cdots=\sigma_{n}(G)=0$. This cannot really happen for graphs, but gives acceptable asymptotics.

Proposition 10 If $p \geq 2$ and $G$ is a graph of order $n$ and size $m$, then

$$
\|G\|_{S_{p}}^{p}<(2 m)^{1 / 2}<n .
$$

For every $m \leq n(n-1) / 2$, there is a graph with $m$ edges satisfying

$$
\|G\|_{S_{p}}^{p}>\left(2 m-O\left(m^{1 / 2}\right)\right)^{1 / 2} .
$$

To see the validity of the last statement, consider the maximal complete graph $K_{s}$ that can be formed with at most $m$ edges. We have $s=\left\lfloor(2 m)^{1 / 2}\right\rfloor$ and

$$
\left\|K_{s}\right\|_{S_{p}}^{p} \geq(s-1)^{p}+s=\left(2 m-O\left(m^{1 / 2}\right)\right)^{1 / 2} .
$$

\subsubsection{A lower bound on Schatten $p$-norms for $p \geq 1$}

In this section we obtain a lower bound on $\|G\|_{S_{p}}$ which extends the bound of Caporossi, Cvetković, Gutman and Hansen [3]

$$
\mathcal{E}(G) \geq 2 \mu_{1}(G),
$$

where equality holds if an only if $G$ is a complete multipartite graph with possibly some isolated vertices. Here the extension to $\|G\|_{S_{p}}$ sheds new light on the original bound (10) by revealing the role of the chromatic number in this relation; obviously, this role is concealed in (10).

Indeed, recall that if $G$ is a graph with chromatic number $\chi$, Hoffman's inequality [14] gives

$$
\left|\mu_{n}(G)\right|+\cdots+\left|\mu_{n-\chi+2}(G)\right| \geq \mu_{1}(G) .
$$

Now we can deduce the following theorem.

Theorem 11 If $p \geq 1$ and $G$ is a graph with chromatic number $\chi$, then

$$
\|G\|_{S_{p}} \geq \sigma_{1}\left(1+(\chi-1)^{1-p}\right)^{1 / p} .
$$

For $p=1$ equality holds if an only if $G$ is a complete $\chi$-partite graph with possibly some isolated vertices. For $p>1$ equality holds if an only if $G$ is a regular complete $\chi$-partite graph with possibly some isolated vertices.

We switch now to the study of the Ky Fan $k$-norms. 


\subsection{The maximal Ky Fan norms for graphs}

In this subsection we study the asymptotics of the maximal Ky Fan $k$-norms for graphs of large order. To approach the problem, let us define the functions $\tau_{k}(n)$ and $\xi_{k}(n)$ as

$$
\begin{aligned}
\tau_{k}(n) & =\max _{v(G)=n} \mu_{1}(G)+\cdots+\mu_{k}(G) \\
\xi_{k}(n) & =\max _{v(G)=n}\|G\|_{F_{k}} .
\end{aligned}
$$

For large $n$ the function $\tau_{k}(n)$ is pretty stable as implied by the main result in [20]:

Theorem 12 For every fixed positive integer $k$, the limit $\tau_{k}=\lim _{n \rightarrow \infty} \tau_{k}(n) / n$ exists.

Following the approach of [20], one can prove an analogous assertion for singular values:

Theorem 13 For every fixed positive integer $k$, the limit $\xi_{k}=\lim _{n \rightarrow \infty} \xi_{k}(n) / n$ exists.

Finding $\tau_{k}(n)$ and $\xi_{k}(n)$ is not easy for any $k \geq 2$, and even finding the limits $\tau_{k}$ and $\xi_{k}$ is challenging. Indeed, even the simplest case $\xi_{2}$ is not known yet, despite intensive research; here is

the story: Gregory, Hershkowitz and Kirkland [10] asked what is the maximal value of the spread of a graph of order $n$, that is to say, what is

$$
\max _{v(G)=n} \mu_{1}(G)-\mu_{n}(G)
$$

This problem is still open, even asymptotically; we will not solve it here, but we will show that it is equivalent to finding

$$
\max _{v(G)=n}\|G\|_{F_{2}} .
$$

Indeed, we have

$$
\|G\|_{F_{2}}=\max \left\{\left|\mu_{1}(G)\right|+\left|\mu_{2}(G)\right|,\left|\mu_{1}(G)\right|+\left|\mu_{n}(G)\right|\right\},
$$

and from [8] it is known that every graph $G$ of order $n$ satisfies

$$
\left|\mu_{1}(G)\right|+\left|\mu_{2}(G)\right| \leq(1 / 2+\sqrt{5 / 12}) n<1.146 n .
$$

On the other hand, in [10], for every $n \geq 2$, a graph $G$ of order $n$ is shown such that

$$
\left|\mu_{1}(G)\right|+\left|\mu_{n}(G)\right| \geq(2 n-1) / \sqrt{3}
$$

hence, $\left|\mu_{1}(G)\right|+\left|\mu_{n}(G)\right|>1.154 n$ for sufficiently large $n$. Therefore, we see that for $n$ large,

$$
\max _{v(G)=n} \mu_{1}(G)-\mu_{n}(G)=\max _{v(G)=n}\|G\|_{F_{2}}=\xi_{2}(n) .
$$




\subsubsection{The asymptotics of $\tau_{k}(n)$ and $\xi_{k}(n)$}

Surprisingly, finding $\tau_{k}$ and $\xi_{k}$ seems a tad easier for large $k$. Indeed, Mohar [19] proved the following bounds

$$
\frac{1}{2}\left(\frac{1}{2}+\sqrt{k}+o\left(k^{-2 / 5}\right)\right) n \leq \tau_{k}(n) \leq \frac{1}{2}(1+\sqrt{k}) n .
$$

In [23], in a different way, we proved the following theorem.

Theorem 14 If $n$ and $k$ are integers such that $n \geq k \geq 2$, then

$$
\frac{1}{2}\left(\frac{1}{2}+\sqrt{k}+o\left(k^{-2 / 5}\right)\right) n \leq \xi_{k}(n) \leq \frac{1}{2}(1+\sqrt{k}) n .
$$

In fact, without extra effort, one can prove a more general upper bound about $(0,1)$-matrices, which we give shortly. To describe the case of equality we need the following definition: a matrix $A$ is called plain if the all one vectors $\mathbf{j}_{m} \in \mathbb{R}^{m}$ and $\mathbf{j}_{n} \in \mathbb{R}^{n}$ are singular vectors to $\sigma_{1}(A)$, that is to say, $\sigma_{1}(A)=\left\langle\mathbf{j}_{m}, A \mathbf{j}_{n}\right\rangle / \sqrt{m n}$.

Theorem 15 Let $n \geq m \geq k \geq 1$ be integers. If $A$ is a $(0,1)$-matrix of size $m \times n$, then

$$
\|A\|_{F_{k}} \leq \frac{1}{2}(1+\sqrt{k}) \sqrt{m n} .
$$

Equality holds in (13) if and only if the matrix $J_{m, n}-2 A$ is plain and has exactly $k$ nonzero singular values which are equal. In particular, if $m=n=k$, equality holds if and only if $J_{m}-2 A$ is a plain Hadamard matrix.

Since for every Hermitian matrix $A$ of size $n \geq k$,

$$
\mu_{1}(A)+\cdots+\mu_{k}(A) \leq\|A\|_{F_{k}}
$$

we see that $\tau_{k}(n) \leq \xi_{k}(n)$, and so the upper bound in Theorem 14 implies Mohar's upper bound. The advantage of Theorem 15 is that this bound is extended to non-square matrices, where eigenvalues are not applicable at all; moreover, along the same lines, Theorem 23 below gives an analogous statement for arbitrary nonnegative matrices. Let us also point out that Theorem 15 neatly proves and generalizes the result of Koolen and Moulton (8) .

There are infinitely many cases when $\xi_{k}(n)$ attains the upper bound of Theorem 14. Indeed, let $k$ be such that there exists a graph $G$ of order $k$ for which the Koolen and Moulton bound (8) is an equality. Then, for every $n \geq 1$, blowing-up $G$ by a coefficient $n$, and calculating the Ky Fan $k$-norm of the resulting graph, we obtain

$$
\xi_{k}(k n)=\frac{1}{2}(1+\sqrt{k}) k n .
$$

In particular, it is known that for $k=4$ the complete graph $K_{4}$ satisfies

$$
\left\|K_{4}\right\|_{F_{4}}=6=\frac{1}{2}(1+\sqrt{4}) 4
$$

and so, $\xi_{4}(4 n)=6 n$ and $\xi_{4}=3 / 2$. Other known $k$ of this type are $k=4 m^{4}$ for all integer $m>1$, (see [13]), but there are also others, like $k=16$ and $k=36$.

Clearly, following the blow-up idea, for infinitely many triples $k, m, n$ one can construct matrices attaining equality in (12). 


\subsection{The Ky Fan norms and chromatic numbers}

Using Hoffman's inequality (11), we obtain another extension of the bound (10):

Theorem 16 If $G$ is a graph with chromatic number $\chi$, then

$$
\|G\|_{F_{\chi}} \geq 2 \sigma_{1}(G) .
$$

Unlike Theorem 11, it is not easy to characterize when equality holds in (13). One possible case is when $G$ is a complete $\chi$-partite graph with some isolated vertices, but there are many other constructions, some of which are rather complicated.

\section{Some bounds on matrix norms}

Recall that an $n \times n$ matrix $A=\left[a_{i j}\right]$ is called Hadamard if $\left|a_{i j}\right|=1$, for all $1 \leq i \leq n, 1 \leq j \leq n$ and $A A^{*}=n I_{n}$. It is known that there are no real Hadamard matrices of some orders, but there are complex Hadamard matrices of any order, for example, the $n \times n$ discrete Fourier transform matrix $A=\left[a_{i j}\right]$ defined by

$$
a_{k j}=\exp (2 \pi i(k-1)(j-1) / n), \quad 1 \leq k \leq n, 1 \leq j \leq n .
$$

Below we will often meet a class of matrices akin to Hadamard matrices: for integral $n \geq m \geq 2$, let $\operatorname{Had}_{m, n}$ denote the class of $m \times n$ matrices whose entries have the same modulus, and whose rows are pairwise orthogonal. Clearly if $A \in \mathrm{Had}_{m, n}$, then its row vectors have the same length, and so $\mathrm{Had}_{n, n}$ is just the set of all scalar multiples of Hadamard matrices. Note that $\operatorname{Had}_{m, n}$ contains complex matrices for any $m$ and $n$, since if $A \in \mathrm{Had}_{n, n}$, then any $m \times n$ submatrix of $A$ belongs to $\operatorname{Had}_{m, n}$.

\subsection{Generalizing McClelland's bound}

Our first goal is to generalize McClelland's bound (5) to arbitrary matrices. Using Jensen's inequality, we immediately obtain the following general bound.

Theorem 17 Let $n \geq m \geq 1$ and let $A \in M_{m, n}$. If $1 \leq p \leq q$, then

$$
m^{-1 / p}\|A\|_{S_{p}} \leq m^{-1 / q}\|A\|_{S_{q}} .
$$

Equality holds in (14) if and only if the rows of $A$ are pairwise orthogonal vectors of equal length.

The last statement can be proved by using the singular value decomposition of $A$ to show that $A A^{*}$ is a scalar multiple of the identity matrix $I_{m}$.

Taking $A$ to be the adjacency matrix of a graph and setting $q=2$, we obtain Proposition 6 , Restricting, in addition, the range of $p$, we obtain an absolute bound on $\|A\|_{S_{p}}$. 
Theorem 18 Let $n \geq m \geq 1$ and let $A \in M_{m, n}$. If $1 \leq p \leq 2$, then

$$
\|A\|_{S_{p}} \leq m^{1 / p} n^{1 / 2}|A|_{\infty} .
$$

Equality holds in (15) if and only if $A \in \operatorname{Had}_{m, n}$.

For arbitrary matrices Theorems 17 and 18 are as good as one can get, but for specific classes of matrices they can be improved further. For instance, if $A$ is a nonzero nonnegative matrix, (14) can be improved as shown in the following subsection.

\subsection{Generalizing the results of Koolen and Moulton}

We start with a fine tuning of (15), which is valid for arbitrary matrices.

Proposition 19 Let $n \geq m \geq 1$ and let $A \in M_{m, n}$. If $1 \leq p \leq q$, then

$$
\|A\|_{S_{p}}^{p} \leq \sigma_{1}^{p}(A)+(m-1)^{1-p / q}\left(\|A\|_{S_{q}}^{q}-\sigma_{1}^{q}(A)\right)^{1 / q} .
$$

Equality in (16) is possible if and only if $\sigma_{2}(A)=\cdots=\sigma_{m}(A)$.

It seems technically difficult to deduce nice acceptable corollaries from (16) for general $p$ and $q$. We shall do this for $p=1$ and $q=2$. In this case we see that

$$
\|A\|_{S_{1}} \leq \sigma_{1}(A)+\sqrt{(m-1)\left(|A|_{2}^{2}-\sigma_{1}^{2}(A)\right)} .
$$

Now, noting that for nonnegative matrices $\sigma_{1}(A) \geq|A|_{1} / \sqrt{m n}$, we get:

If $n \geq m \geq 1$ and $A$ is an $m \times n$ nonnegative matrix with $|A|_{1} \geq n|A|_{\infty}$, then

$$
\|A\|_{S_{1}} \leq \frac{|A|_{1}}{\sqrt{m n}}+\sqrt{(m-1)\left(|A|_{2}^{2}-\frac{|A|_{1}^{2}}{m n}\right)} \leq \frac{(m+\sqrt{m}) \sqrt{n}}{2}|A|_{\infty} .
$$

Inequalities (17) and (18) were given first in 21]; obviously they extend the corresponding inequalities of Koolen and Moulton [17] for graphs. One question that has been omitted in [21] is: When do we have the equality

$$
\|A\|_{S_{1}}=\frac{(m+\sqrt{m}) \sqrt{n}}{2}|A|_{\infty} .
$$

For simplicity assume that $|A|_{\infty}=1$. Analyzing the proof in [21], one can deduce that $A$ must be a $(0,1)$-matrix with

$$
\sigma_{1}(A)=\frac{(1+\sqrt{m}) \sqrt{n}}{2}, \sigma_{2}(A)=\cdots=\sigma_{m}(A)=\frac{\sqrt{n}}{2} .
$$

After some calculations, we find that $|A|_{1}=(m+\sqrt{m}) n / 2$, and $A$ has equal column sums and equal row sums. For some values of $m$ and $n$ it is possible to construct matrices satisfying (19). For instance, let $B$ be the adjacency matrix of a graph of order $m$ having maximum energy $(1+\sqrt{m}) m / 2$. Consider the $m \times 2 m$ block matrix $A=(B, B)$. After some algebra, we see that $A$ satisfies (19).

It turns out that nonnegative matrices satisfying (19) can be characterized in another way 
Theorem 20 Let $n \geq m \geq 1$. A nonnegative $m \times n$ matrix A satisfies

$$
\|A\|_{S_{1}}=\frac{(m+\sqrt{m}) \sqrt{n}}{2}|A|_{\infty}
$$

if and only if the matrix $2 A-J_{m, n}$ is plain and belongs to $\operatorname{Had}_{m, n}$.

\subsection{The maximal Ky Fan norms of matrices}

Here, the aim is to extend Theorem 14 to arbitrary matrices. Using the Cauchy- Schwarz inequality, we obtain the following theorem.

Theorem 21 Let $n \geq m \geq k \geq 1$. If $A \in M_{m, n}$ then

$$
\|A\|_{F_{k}} \leq \sqrt{k}|A|_{2} .
$$

Equality holds for infinitely many types of matrices.

To prove the last statement, let $q \geq k$ and let $B \in$ be a $k \times q$ matrix whose rows are pairwise orthogonal vectors of equal length, that is to say, all $k$ singular values of $B$ are equal. Writing $\otimes$ for the Kronecker product and setting $A=B \otimes J_{r, s}$, we see that $A$ has exactly $k$ nonzero singular values, which are equal, and so $\|A\|_{F_{k}} \leq \sqrt{k}|A|_{2}$.

Theorem 22 Let $n \geq m \geq k \geq 1$. If $A \in M_{m, n}$ then

$$
\|A\|_{F_{k}} \leq \sqrt{k m n}|A|_{\infty} .
$$

Equality holds for infinitely many types of matrices.

As above, to prove the last statement, let $q \geq k$, let $B \in \operatorname{Had}_{k, q}$, and set $A=B \otimes J_{r, s}$. Since $A$ has exactly $k$ nonzero singular values, which are equal, and since all entries of $A$ are equal in absolute value, we have $\|A\|_{F_{k}}=\sqrt{k}|A|_{2}=\sqrt{k m n}|A|_{\infty}$, as claimed.

These bounds are as good as one can get, but for nonnegative matrices there is a slight improvement.

Theorem 23 Let $n \geq m \geq k \geq 1$. If $A \in M_{m, n}$ is a nonnegative matrix then then

$$
\|A\|_{F_{k}} \leq \frac{1}{2}(1+\sqrt{k}) \sqrt{m n}|A|_{\infty}
$$

Equality holds in (19) if and only if $A$ is a scalar multiple of a $(0,1)$-matrix, the matrix $|A|_{\infty}\left(J_{m, n}-2 A\right)$ is plain and has exactly $k$ nonzero singular values which are equal.

\section{Some open problems}

(1) Find the extrema of $\|G\|_{S_{p}}$ and $\|G\|_{F_{k}}$ when $G$ belongs to a given monotone or hereditary property.

(2) Same problem for matrices.

(3) Find the best approximation of $\max \left\{\|G\|_{S_{p}}: v(G)=n\right\}$ for all $n$ and all $1 \leq p \leq 2$.

(4) Find necessary and sufficient conditions for equality in Theorems 21, 22, and 23, 


\section{References}

[1] L. Arnold, On the asymptotic distribution of the eigenvalues of random matrices, J. Math. Anal. Appl. 20 (1967) 262-268.

[2] B. Bollobás, Modern Graph Theory, Graduate Texts in Mathematics, 184, Springer-Verlag, New York (1998), xiv+394 pp.

[3] G. Caporossi, D. Cvetković, I. Gutman, P. Hansen, Variable neighborhood search for extremal graphs. 2. Finding graphs with extremal energy, J. Chem. Inf. Comput. Sci. 39 (1999), 984-996.

[4] J. Day and W. So, Singular value inequality and graph energy change, Electron. J. Linear Algebra 16 (2007), 291-299.

[5] J. Day and W. So, Graph energy change due to edge deletion, Linear Algebra Appl. 428 (2008), 2070-2078.

[6] W. Du, X. Li and Y. Li, The Laplacian energy of random graphs, J. Math. Anal. Appl. 368 (2010) 311-319.

[7] W. Du, X. Li and Y. Li, The energy of random graphs, to appear in Linear Algebra Appl.

[8] J. Ebrahimi, B. Mohar, V. Nikiforov, and A.S. Ahmady, On the sum of two largest eigenvalues of a symmetric matrix, Linear Algebra Appl. 429 (2008), 2781-2787.

[9] Z. Füredi and J. Komlós, The eigenvalues of random symmetric matrices, Combinatorica 1 (1981), 233-241.

[10] D. Gregory, D. Hershkowitz and S. Kirkland, The spread of the spectrum of a graph, Linear Algebra Appl. 332 (2001), 23-35.

[11] I. Gutman, The energy of a graph, Ber. Math.-Stat. Sekt. Forschungszent. Graz 103 (1978), $1-22$.

[12] I. Gutman, The energy of a graph: old and new results, Algebraic Combinatorics and Applications (Gössweinstein, 1999), Springer, Berlin, 2001, pp. 196-211.

[13] W. Haemers and Q. Xiang, Strongly regular graphs with parameters $\left(4 m^{4}, 2 m^{4}+m^{2}, m^{4}+m^{2}\right.$, $m^{4}+m^{2}$ ) exist for all $m>1$, to appear in Eur. J. Combin.

[14] A.J. Hoffman, On eigenvalues and colorings of graphs, Graph Theory and its Applications, Academic Press, New York (1970), pp. 79-91.

[15] H. Dwight, Tables of Integrals and Other Mathematical Data, 3rd ed., Macmillan, 1957.

[16] R. Horn and C. Johnson, Matrix Analysis, Cambridge University Press, Cambridge, 1985, xiii+561 pp.

[17] J.H. Koolen and V. Moulton, Maximal energy graphs, Adv. Appl. Math. 26 (2001), 47-52. 
[18] B. McClelland, Properties of the latent roots of a matrix: The estimation of $\pi$-electron energies, J. Chem. Phys. 54 (1971), 640-643.

[19] B. Mohar, On the sum of $k$ largest eigenvalues of graphs and symmetric matrices, J. Combin. Theory, Ser. B 99 (2009), 306-313.

[20] V. Nikiforov, Linear combinations of graph eigenvalues, Electron. J. Linear Algebra 15 (2006), 329-336.

[21] V. Nikiforov, The energy of graphs and matrices, J. Math. Anal. Appl. 326 (2007) 1472-1475.

[22] V. Nikiforov, Graphs and matrices with maximal energy, J. Math. Anal. Appl. 327 (2007), 735-738.

[23] V. Nikiforov, On the sum of $k$ largest singular values of graphs and matrices, submitted for publication; preprint ava4ilable at arXiv:1007.3949.

[24] W. So, M. Robbiano, N. de Abreu and I. Gutman, Applications of a theorem by Ky Fan in the theory of graph energy, Linear Algebra Appl. 432, (2010), 2163-2169.

[25] E. Wigner, On the Distribution of the Roots of Certain Symmetric Matrices, Ann. of Math. 67 (1958), 325-328. 